\title{
ADVANCES IN APPLIED PROBABILITY \\ VOLUME 39 (2007): INDEX \\ Stochastic Geometry and Statistical Applications
}

Averkov, G. AND Bianchi, G. Retrieving convex bodies from restricted covariogram functions ..

BALLANI, F. The surface pair correlation function for stationary Boolean models .............

Baumstark, V. and Last, G. Some distributional results for Poisson-Voronoi tessellations .......

BiAnchi, G. see Averkov, G.

Borovkov, K. A. AND Odell, D. A. On spatial thinning-replacement processes based on Voronoi

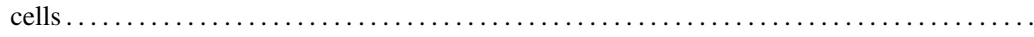

CowAN, R. Identities linking volumes of convex hulls $\ldots \ldots \ldots \ldots \ldots \ldots \ldots \ldots \ldots \ldots \ldots \ldots \ldots \ldots \ldots \ldots$

Ganesh, A. AND XuE, F. On the connectivity and diameter of small-world networks..........

Hoffmann, L. M. Intersection densities of nonstationary Poisson processes of hypersurfaces .....

On weak stationarity and weak isotropy of processes of convex bodies and cylinders........

LAST, G. see BAUMSTARK, V.

MÅnsson, M. A connection between the volume fractions of the Stienen model and the dead leaves model . .

PAGES

613-629

$1-15$

$16-40$

Odell, D. A. see Borovkov, K. A.

Olsbo, V. On the correlation between the volumes of the typical Poisson-Voronoi cell and the typical Stienen sphere.

Pimentel, L. P. R. Multitype shape theorems for first passage percolation models ............

Roy, E. Bartlett spectrum and mixing properties of infinitely divisible random measures .........

Thorisson, H. The Palm-duality for random subsets of $d$-dimensional grids $\ldots \ldots \ldots \ldots \ldots \ldots$

WADE, A. R. Explicit laws of large numbers for random nearest-neighbour type graphs .........

Xue, F. see GANESH, A. 